NBER WORKING PAPER SERIES

\title{
RISK AVERSION AND OPTIMAL PORTFOLIO POLICIES IN PARTIAL AND GENERAL EQUILIBRIUM ECONOMIES
}

\author{
Leonid Kogan \\ Raman Uppal \\ Working Paper 8609 \\ http://www.nber.org/papers/w8609 \\ NATIONAL BUREAU OF ECONOMIC RESEARCH \\ 1050 Massachusetts Avenue \\ Cambridge, MA 02138 \\ November 2001
}

Financial support from the Rodney L. White Center for Financial Research is gratefully acknowledged. We are grateful to Bernard Dumas for detailed suggestions on the manuscript. We would also like to acknowledge comments from Harjoat Bhamra, John Campbell, George Chacko, Lorenzo Garlappi, Francisco Gomes, Mark Grinblatt, Denis Gromb, Ulrich Haussmann, Claus Munk, Vasant Naik, Jiang Wang, Tan Wang and seminar participants at Boston College,London Business School,London School of Economics, UBC Commerce, the UBC Pacific Institute for Mathematical Sciences, the Wharton Lunch Seminar Series, UCLA, University of Vienna, SIRIF Conference on Dynamic Portfolio Strategies, TMR-HERMES Conference on Savings, Portfolios and Pensions, and the Western Finance Association Meetings (Sun Valley,2000). The views expressed herein are those of the authors and not necessarily those of the National Bureau of Economic Research.

(C) 2001 by Leonid Kogan and Raman Uppal. All rights reserved. Short sections of text, not to exceed two paragraphs, may be quoted without explicit permission provided that full credit, including $(\mathrm{C}$ notice, is given to the source. 
Risk Aversion and Optimal Portfolio Policies in

Partial and General Equilibrium Economies

Leonid Kogan and Raman Uppal

NBER Working Paper No. 8609

November 2001

JEL No. G12, G11, D52, C63

\section{ABSTRACT}

In this article, we show how to analyze analytically the equilibrium policies and prices in an economy with a stochastic investment opportunity set and incomplete financial markets, when agents have power utility over both intermediate consumption and terminal wealth, and face portfolio constraints. The exact local comparative statics and approximate but analytical expression for the portfolio policy and asset prices are obtained by developing a method based on perturbation analysis to expand around the solution for an investor with log utility. We then use this method to study a general equilibrium exchange economy with multiple agents who differ in their degree of risk aversion and face borrowing constraints. We characterize explicitly the consumption and portfolio policies and also the properties of asset returns. We find that the volatility of stock returns increases with the cross-sectional dispersion of risk aversion, with the cross-sectional dispersion in portfolio holdings, and with the relaxation of the constraint on borrowing. Moreover, tightening the borrowing constraint lowers the riskfree interest rate and raises the equity premium in equilibrium.

Leonid Kogan

Sloan School of Management

Massachusetts Institute of Technology

50 Memorial Drive, E52-455

Cambridge, MA 02142

and NBER

Tel: 617-253-2289

Email: 1kogan@mit.edu
Raman Uppal

London Business School

6 Sussex Place

Regent's Park, London

United Kingdom

NW1 4SA

Tel: 44-20-7706-6883

Email: ruppal@london.edu 


\section{Introduction}

Merton $(1969,1971)$ shows that in an environment where investment opportunities vary over time, investors optimizing over a single period will choose portfolios that are different from investors optimizing over multiple periods. This is because the optimal intertemporal portfolio is not necessarily instantaneously mean-variance efficient, but also provides a hedge against future shifts in the investment opportunity set. ${ }^{1}$ However, these papers do not indicate how one can obtain explicit solutions: in the dynamic programming formulation of Merton, obtaining an explicit solution requires one to solve a nonlinear partial differential equation for which a closed-form solution is typically not available.

To obtain explicit solutions to the non-linear differential equation characterizing the intertemporal portfolio problem, research building on the work of Merton has proceeded in three directions. One strand - for example, Liu (1998) and Wachter (1998) in partial equilibrium and Wang (1996) in general equilibrium - assumes that financial markets are complete, and then uses the martingale technique of Cox and Huang (1989) to determine the optimal consumption and portfolio rules in two distinct steps: first, consumption is identified by solving a static optimization problem, and then the optimal portfolio rules are obtained by solving a linear differential equation. A second strand - for instance, Kim and Omberg (1996) and Liu (1998) — assumes that investors derive utility only from terminal wealth and not from intermediate consumption, which is one of the sources of the non-linearity in the differential equation. Then, under a particular specification of the investment opportunity set, this assumption allows them to solve the dynamic programming equation in closed form. A third approach, developed by Campbell (1993), allows for intermediate consumption and incomplete financial markets, but chooses a convenient specification for the evolution of the investment opportunity set, and then makes appropriate approximations in order to overcome the non-linearity of the problem. ${ }^{2}$ Thus, the first approach needs to make restrictive assumptions about the structure of financial markets, while the other two approaches work only for particular specifications for the evolution of the investment opportunity set; moreover, all three approaches

\footnotetext{
${ }^{1}$ The static and dynamic portfolios will coincide only under specific conditions for the utility function (unit risk aversion) or asset returns (independence between changes in the investment opportunity and asset returns). Results in the empirical literature suggest that it is unlikely that either condition is true; a discussion of this literature is in Campbell and Viceira (1999).

${ }^{2}$ For models set in discrete time one needs to log-linearize the budget equation and the first-order conditions, and for models set in continuous time one needs to log-linearize the Hamilton-Jacobi-Bellman equation.
} 
are not suitable when there are constraints on portfolio positions.

This article contributes to the literature on portfolio choice and asset pricing in two ways. First, we develop a method to analyze analytically the equilibrium policies and prices in an economy with a stochastic investment opportunity set and incomplete financial markets, when agents have power utility over both intermediate consumption and terminal wealth. This method can be applied to characterize portfolio policies in partial-equilibrium models where the risk-free interest rate, the expected stock return and the volatility of stock returns can change over time. This method can also be used to study general equilibrium economies with portfolio constraints when there are multiple investors who differ in their risk aversion, and hence, the investment opportunity set evolves endogenously.

Our second contribution is to apply this method to a particular general equilibrium setting in order to understand the effect on asset prices of portfolio constraints and of heterogeneity. We study a general equilibrium exchange economy with an arbitrary number of agents who differ in their degree of risk aversion and face borrowing constraints. Our main findings are that the volatility of stock returns increases with the cross-sectional dispersion of risk aversion, with the cross-sectional dispersion of portfolio holdings, and as one relaxes the constraint on borrowing. Moreover, tightening the borrowing constraint lowers the risk-free interest rate and raises the equity premium in equilibrium.

Our analytical characterization of portfolio policies and prices in economies with an arbitrary number of agents, who differ in their risk aversion and face borrowing constraints, extends the analysis of a production economy in Dumas (1989) and of an exchange economy in Wang (1996). In contrast to our work, both these papers assume that financial markets are complete and do not consider the case of portfolio constraints; moreover, the model in Dumas can be solved only numerically even when there are only two agents, while Wang can solve for only some of the quantities of the model in closed form and even this is possible only for particular permutations of the number of agents and the degree of risk aversion for each of these agents.

Several papers study economies in which agents have heterogeneous preferences in the presence of portfolio constraints. Cuoco (1997) characterizes the risk premium in an economy where agents differ with respect to their risk aversion face portfolio constraints. Detemple and Murthy (1997) 
also study a heterogeneous-agent general equilibrium model with portfolio constraints where the heterogeneity arises from differences in beliefs rather than differences in risk aversion (all agents have $\log$ utility); they show that some of their results on pricing would extend to an economy where agents differ in risk aversion, but do not provide an explicit characterization of optimal policies. Marcet and Singleton (1999) use simulation methods to analyze an economy where agents face borrowing constraints and differ with respect to their labor income and risk aversion. In contrast to these papers, we provide an explicit characterization in terms of exogenous variables for the consumption and portfolio policies, the riskless rate and the stock price, and also for the mean and volatility of stock returns.

Identifying the equilibrium in multiagent economies with incomplete financial markets is a difficult problem and to date the literature does not have an explicit general characterization in terms of exogenous variables. Cuoco and He (1994a,b) show that with incomplete markets one can still construct a representative agent, but in this case the weights assigned to individual agents in this aggregation evolve stochastically. However, their characterization of equilibrium is in terms of endogenous variables. Our approach can be viewed as a convenient way of expressing the solution in terms of the primitives in economies where financial markets are incomplete and agents differ in their degree of risk aversion.

Our method relies on asymptotic analysis, which allows one to obtain in closed-form the approximate (asymptotic) expressions for portfolio and consumption policies. The basic idea of asymptotic methods is to formulate a general problem, find a particular case that has a known solution, and use this as a starting point for computing the solution to nearby problems. In the context of portfolio problems, the solution for the investor with log utility (with unit risk aversion) provides a convenient starting point for the expansion. We need to emphasize, though, that while our method allows for exact comparative statics results around the case of log utility, it provides only approximations to the portfolio rules and asset prices, and thus, it should be viewed as being complementary to numerical methods rather than a substitute. While the asymptotic solution is designed to provide a local approximation (for risk aversion close to unity), general theoretical results on the magnitude of the approximation error are currently not available - see Judd (1996, 1998, Ch. 13-15) for a discussion of these issues. However, there are a number of methods to evaluate the quality of the approximate solution numerically (for instance, see Den Haan and Marcet, 1994, and Judd, 1996 
and 1998) that can also be applied to the portfolio problems considered here.

The rest of the paper is arranged as follows. In Section 2, we describe the method for analyzing portfolio decisions in the context of an arbitrary vector process driving investment opportunities. In Section 3, we apply this method to a particular general equilibrium exchange economy where investors vary in their degree of risk aversion and face leverage constraints. We conclude in Section 4. The main results of each section are highlighted in propositions and the proofs for all the propositions are collected in the appendix.

\section{An asymptotic approach to consumption and portfolio choice}

In this section, we undertake an asymptotic analysis of a model of consumption and portfolio selection with a stochastic investment opportunity set, when the agent derives utility from intermediate consumption and bequest, and faces constraints on her portfolio position. We show that one can obtain an explicit asymptotic expression for the solution of the intertemporal consumption-portfolio problem, as long as the value function of the analogous problem for the agent with logarithmic preferences is known in closed form.

The section is structured as follows. We start by describing a partial-equilibrium economy with an arbitrary stochastic vector process for the state variables that drives changes in the investment opportunity set. Following this, we first derive the consumption and portfolio rules in the absence of constraints and then consider the effect of constraints on portfolio positions. We conclude by discussing how these results can be extended to a general equilibrium setting.

\subsection{The economy}

In this section, we describe the features of the model: the preferences of agents, the financial assets that they can choose to hold, and the stochastic nature of the investment opportunity set. 


\subsubsection{Preferences}

The utility function of the agent is time-separable and is given by

$$
\psi \cdot \mathrm{E}_{0}\left[\int_{0}^{T} e^{-\rho t} \frac{1}{\gamma}\left(C_{t}^{\gamma}-1\right) d t\right]+(1-\psi) \cdot e^{-\rho T} \mathrm{E}_{0}\left[\frac{1}{\gamma}\left(W_{T}^{\gamma}-1\right)\right]
$$

where $\rho$ is the constant subjective time discount rate, $C_{t}$ is the flow of consumption, and the preference parameter $\psi$ controls the relative weight of intermediate consumption and the end-ofperiod wealth (bequest) in the agent's utility function. The agent's relative risk aversion is given by $1-\gamma$, and for agents with unit risk aversion $(\gamma=0)$, utility is given by the logarithmic function:

$$
\psi \cdot \mathrm{E}_{0}\left[\int_{0}^{T} e^{-\rho t} \ln C_{t} d t\right]+(1-\psi) \cdot e^{-\rho T} \mathrm{E}_{0}\left[\ln W_{T}\right]
$$

\subsubsection{Financial assets}

The agent can allocate her wealth to two assets: a short-term riskless asset (bond) with rate of return $r_{t}$, and a stock (paying zero dividend). ${ }^{3}$ The price of the stock, $P_{t}$, evolves according to

$$
\frac{d P_{t}}{P_{t}}=\mu_{P t} d t+\sigma_{P t} d Z_{P t}
$$

where $\mu_{P t}$ is the instantaneous expected return and $\sigma_{P t}$ is the volatility. Our convention is to denote stochastic variables with a subscript " $t$ "; thus, in the above specification, the riskless rate, $r_{t}$, the expected return on the stock, $\mu_{P t}$, and the volatility of stock returns, $\sigma_{P t}$, are permitted to be stochastic.

\subsubsection{The investment opportunity set}

The investment opportunity set is described by the vector of state variables, $\mathbf{X}_{t}$. The state vector is assumed to change over time according to

$$
d \mathbf{X}_{t}=\mu_{\mathbf{X}}\left(\mathbf{X}_{t}\right) d t+\sigma_{\mathbf{X}}^{\prime}\left(\mathbf{X}_{t}\right) \cdot d \mathbf{Z}_{\mathbf{X} t}
$$

where the covariance between the stock returns process and the state vector process is denoted by $\sigma_{P \mathbf{X}}$. With the above specification, the riskless rate and the expected rate of return and volatility

\footnotetext{
${ }^{3}$ The extension to multiple risky assets is straightforward.
} 
of the risky asset may depend on the state vector:

$$
r_{t}=r\left(\mathbf{X}_{t}\right), \quad \mu_{P t}=\mu_{P}\left(\mathbf{X}_{t}\right), \quad \sigma_{P t}=\sigma_{P}\left(\mathbf{X}_{t}\right)
$$

implying that the instantaneous market price of risk is also stochastic:

$$
\phi_{t}=\phi\left(\mathbf{X}_{t}\right) \equiv \frac{\mu_{P t}-r_{t}}{\sigma_{P t}^{2}}
$$

\subsection{Consumption and portfolio policies in the absence of portfolio constraints}

In the above economy, denoting by $\pi_{t}$ the proportion of the agent's wealth invested in the risky asset, the wealth of the agent evolves according to

$$
d W_{t}=\left[\left(r_{t}+\pi_{t}\left(\mu_{P t}-r_{t}\right)\right) W_{t}-C_{t}\right] d t+\pi_{t} \sigma_{P t} W_{t} d Z_{P_{t}}
$$

The value function $J(W, \mathbf{X}, t)$ of the optimal control problem is defined by

$$
J\left(W_{t}, \mathbf{X}_{t}, t\right)=\sup _{\left\{C_{s}, \pi_{s}\right\}} \psi \cdot \mathrm{E}_{t}\left[\int_{t}^{T} e^{-\rho(s-t)} \frac{1}{\gamma}\left(C_{s}^{\gamma}-1\right) d s\right]+(1-\psi) \cdot e^{-\rho(T-t)} \mathrm{E}_{t}\left[\frac{1}{\gamma}\left(W_{T}^{\gamma}-1\right)\right]
$$

subject to equations (1), (2), and (3). Defining the consumption-wealth ratio $c \equiv C / W$, the function $J(W, \mathbf{X}, t)$, satisfies the Hamilton-Jacobi-Bellman equation

$$
0=\max _{c, \pi}\left\{\begin{array}{c}
\frac{\psi}{\gamma}\left((W c)^{\gamma}-1\right)+J_{t}-\rho J+\left(r+\pi\left(\mu_{P t}-r_{t}\right)-c\right) J_{W} W+\frac{1}{2} \pi^{2} W^{2} J_{W W} \sigma_{P}^{2} \\
+\mu_{\mathbf{X}}^{\prime} \cdot J_{\mathbf{X}}+\frac{1}{2} \sigma_{\mathbf{X}}^{\prime} \cdot J_{\mathbf{X X}} \cdot \sigma_{\mathbf{X}}+\pi W \sigma_{P \mathbf{X}}^{\prime} \cdot J_{W \mathbf{X}}
\end{array}\right\}
$$

Given the homogeneity of the utility function, the solution to this equation has the following functional form:

$$
J(W, \mathbf{X}, t)=\frac{A(t)}{\gamma}\left(\left(e^{g(\mathbf{X}, t)} W\right)^{\gamma}-1\right)
$$

where

$$
A(t)=\left(1-\psi \frac{1+\rho}{\rho}\right) e^{-\rho(T-t)}+\frac{\psi}{\rho} .
$$

The exact solution for the optimal consumption policy and portfolio weight can be obtained from 
the first-order conditions implied by the Hamilton-Jacobi-Bellman equation:

$$
\begin{aligned}
c(\mathbf{X}, t) & =\left(\frac{1}{\psi} A(t) e^{\gamma g(\mathbf{X}, t)}\right)^{1 /(\gamma-1)}, \\
\pi(\mathbf{X}, t) & =-\frac{J_{W}}{W J_{W W}} \phi(\mathbf{X})-\frac{J_{W}}{W J_{W W}} \frac{J_{W \mathbf{X}}}{J_{W}} \frac{\sigma_{P \mathbf{X}}^{\prime}(\mathbf{X})}{\sigma_{P}^{2}(\mathbf{X})} \\
& =\frac{1}{1-\gamma} \phi(\mathbf{X})+\frac{\gamma}{1-\gamma} \frac{\sigma_{P \mathbf{X}}^{\prime}(\mathbf{X})}{\sigma_{P}^{2}(\mathbf{X})} \frac{\partial g(\mathbf{X}, t)}{\partial \mathbf{X}}
\end{aligned}
$$

where the second line is obtained by using (4).

In general, the unknown function $g(\mathbf{X}, t)$ cannot be computed in closed form. Our approach is to obtain an asymptotic approximation to $g(\mathbf{X}, t)$, where the expansion is with respect to the risk aversion parameter, $\gamma$. In order to use our results also in a general equilibrium setting with multiple agents who differ in their risk aversion, we define $\gamma \equiv \epsilon a$, where $a$ is used to index agent types so that differences in $a$ lead to differences in risk aversion, while the parameter $\epsilon$ allows us to set the magnitude of these differences. With the above specification, we look for $g(\mathbf{X}, t)$ as a power series in $\epsilon$ :

$$
g(\mathbf{X}, t)=g_{0}(\mathbf{X}, t)+\epsilon g_{1}(\mathbf{X}, t)+O\left(\epsilon^{2}\right)
$$

where $g_{0}(\mathbf{X}, t)$ is obtained from the value function of an agent with logarithmic utility $(\epsilon=0)$ :

$$
J(W, \mathbf{X}, t)=A(t)\left(\ln W(t)+g_{0}(\mathbf{X}, t)\right)
$$

Note that the first-order asymptotic expansions are sufficient to obtain exact local comparative statics results for the dependence of the optimal policies on the risk aversion parameter. The asymptotic expansions will also approximate the optimal consumption and portfolio policies when the risk aversion parameter $\gamma$ is sufficiently close to zero (that is, when $\epsilon$ is close to zero).

We now derive the asymptotic expansions for the consumption-portfolio problem (by substituting (6) into (5)) and explain how one can obtain the function $g_{0}(\mathbf{X}, t)$. Following this, we examine the comparative statics properties of the optimal policies.

Proposition 2.1 The first-order asymptotic expansions for the optimal consumption and portfolio 
choice are

$$
\begin{aligned}
c(\mathbf{X}, t) & =\frac{\psi}{A(t)}-\epsilon a \frac{\psi}{A(t)}\left(g_{0}(\mathbf{X}, t)+\ln \left(\frac{A(t)}{\psi}\right)\right)+O\left(\epsilon^{2}\right), \\
\pi(\mathbf{X}, t) & =\frac{1}{1-\epsilon a} \phi(\mathbf{X})+\frac{\epsilon a}{1-\epsilon a} \frac{\sigma_{P \mathbf{X}}^{\prime}(\mathbf{X})}{\sigma_{P}^{2}(\mathbf{X})} \frac{\partial g_{0}(\mathbf{X}, t)}{\partial \mathbf{X}}+O\left(\epsilon^{2}\right) .
\end{aligned}
$$

An asymptotically equivalent expression for the portfolio choice is given by

$$
\pi(\mathbf{X}, t)=\phi(\mathbf{X})+\epsilon a\left(\frac{\phi(\mathbf{X})}{\sigma_{P}(\mathbf{X})}+\frac{\sigma_{P \mathbf{X}}^{\prime}(\mathbf{X})}{\sigma_{P}^{2}(\mathbf{X})} \frac{\partial g_{0}(\mathbf{X}, t)}{\partial \mathbf{X}}\right)+O\left(\epsilon^{2}\right)
$$

where the function $g_{0}(\mathbf{X}, t)$ is

$$
\begin{aligned}
g_{0}(\mathbf{X}, t)= & \psi \ln \psi \frac{1-e^{-\rho(T-t)}}{\rho A(t)}-\psi \frac{1}{A(t)} \int_{t}^{T} e^{-\rho(s-t)} \ln A(s) d s+ \\
& \frac{1}{A(t)} \mathrm{E}_{t}\left[\int_{t}^{T}\left(A(t)-\frac{\psi}{\rho}\left(1-e^{-\rho(s-t)}\right)\right)\left(-\frac{\psi}{A(s)}+r\left(\mathbf{X}_{s}\right)+\frac{\phi\left(\mathbf{X}_{s}\right)^{2} \sigma_{P}\left(\mathbf{X}_{s}\right)^{2}}{2}\right) d s\right] .
\end{aligned}
$$

The two expressions for the portfolio weight, (8) and (9), are equally easy to manipulate. The role of the risk aversion coefficient is more apparent in (9), while (8) retains the exact form of the myopic portfolio demand, expanding only the hedging demand.

Comparing the asymptotic weight in (8) to the exact one in (5), we see that the only difference is that under the standard approach one needs to identify the unknown function $g(\mathbf{X}, t)$, while in our approach one needs to identify only $g_{0}(\mathbf{X}, t)$, the value function for the log investor. It is much easier to solve for the value function of the log investor. The intuition for this is well-known: ${ }^{4}$ the substitution effect and the income effect arising from a change in the investment opportunity set are of exactly the same magnitude and opposite sign for an investor with log utility. Consequently, this investor has zero demand for hedging future changes in the investment opportunity set, and so her portfolio coincides with the myopic portfolio. Similarly, log-utility investors do not adjust their consumption-wealth ratio for changes in the investment opportunity set, and so it is easy to identify this ratio as a deterministic function of time, $1 / A(t)$. As long as the function $g_{0}(\mathbf{X}, t)$ is known in closed form, one can obtain explicit first-order asymptotic expressions for the optimal

\footnotetext{
${ }^{4}$ Early results on the properties of the log utility function are in Leland (1968) and Mossin (1968). These results were developed further in Hakansson (1971) and Merton (1971).
} 
consumption and portfolio policies. For example, the class of affine processes will yield closed-form solutions.

Analyzing the consumption-portfolio rules given in Proposition 2.1, we see that the zero-order components of these expansions correspond to the well-known solution for the case where the agent has a logarithmic utility function $(\epsilon=0)$ : the optimal consumption-wealth ratio, $c \equiv C / W$, is given by the deterministic function $1 / A(t)$, and the optimal portfolio policy is myopic and independent of changes in the investment opportunity set. The first-order terms capture the effect of risk aversion when the coefficient of relative risk aversion deviates from one ( $\epsilon$ deviates from zero). In particular, one can interpret the expression for the optimal portfolio in (8) as

$$
\pi(\mathbf{X}, t)=\underbrace{\frac{1}{1-\epsilon a} \phi(\mathbf{X})}_{\text {myopic demand }}+\underbrace{\frac{\epsilon a}{1-\epsilon a}\left(\frac{1}{\sigma_{P}^{2}} \sigma_{P \mathbf{X}}^{\prime} \cdot \frac{\partial g_{0}(\mathbf{X}, t)}{\partial \mathbf{X}}\right)}_{\text {hedging demand }}+O\left(\epsilon^{2}\right)
$$

where the first bracketed term represents the portfolio weights under constant investment opportunity set, the myopic demand, and the second term characterizes the demand arising from the desire to hedge against changes in the investment opportunity set. The important thing to note in the above expression is that it relies on $g_{0}$, which can be determined explicitly, rather than on $g$, which cannot be identified generally.

The equation above allows one to obtain the intuitive comparative static results: the hedging demand is asymptotically proportional to the risk aversion parameter and vanishes as $\epsilon$ approaches zero. The hedging demand is also proportional to the scalar product of the vector of "betas" of the state variables with respect to the risky asset, $\sigma_{P}^{-2} \sigma_{P \mathbf{X}}^{\prime}$, and the "delta" of the function $g_{0}(\mathbf{X}, t)$ with respect to the state vector, $\partial g_{0}(\mathbf{X}, t) / \partial \mathbf{X}$. Finally, the equation shows that the hedging demand is zero when the shocks to the state variables are uncorrelated with the returns on the stock $\left(\sigma_{P \mathbf{X}}=0\right)$.

The asymptotic expansions (7) and (9) approximate the optimal consumption and portfolio policies when the risk aversion parameter $\epsilon$ is sufficiently close to zero. They also provide exact local comparative static results for the dependence of the optimal policies on the risk aversion 
parameter:

$$
\begin{aligned}
& \left.\frac{\partial c(\mathbf{X}, t)}{\partial \epsilon}\right|_{\epsilon=0}=a\left[\frac{-\psi}{A(t)}\left(g_{0}(\mathbf{X}, t)+\ln \left(\frac{A(t)}{\psi}\right)\right)\right] \\
& \left.\frac{\partial \pi(\mathbf{X}, t)}{\partial \epsilon}\right|_{\epsilon=0}=a\left[\phi(\mathbf{X})+\frac{1}{\sigma_{P}^{2}(\mathbf{X})} \sigma_{P \mathbf{X}}^{\prime}(\mathbf{X}) \cdot \frac{\partial g_{0}(\mathbf{X}, t)}{\partial \mathbf{X}}\right] .
\end{aligned}
$$

Equation (11) indicates that the optimal position in the risky asset can either increase or decrease with the risk aversion coefficient, depending on the magnitude of the second term in equation (11), which is the sensitivity of the hedging demand with respect to the parameter $\epsilon$.

Infinite-horizon economies are a special case of the general formulation of the previous section. Because of the importance of infinite-horizon models, we present the result for this case below as a separate proposition, which can be obtained from Proposition 2.1 by setting $\psi=1$ and taking the limit as $T \rightarrow \infty$.

Proposition 2.2 The first-order asymptotic expansions for the optimal consumption and portfolio choice are

$$
\begin{aligned}
c(\mathbf{X}) & =\rho-\epsilon a \rho\left(g_{0}(\mathbf{X})-\ln (\rho)\right)+O\left(\epsilon^{2}\right), \\
\pi(\mathbf{X}) & =\frac{1}{1-\epsilon a} \phi(\mathbf{X})+\frac{\epsilon a}{1-\epsilon a} \frac{\sigma_{P \mathbf{X}}^{\prime}(\mathbf{X})}{\sigma_{P}^{2}(\mathbf{X})} \frac{\partial g_{0}(\mathbf{X})}{\partial \mathbf{X}}+O\left(\epsilon^{2}\right),
\end{aligned}
$$

with an asymptotically equivalent expression for the portfolio choice being

$$
\pi(\mathbf{X})=\phi(\mathbf{X})+\epsilon a\left(\phi(\mathbf{X})+\frac{\sigma_{P \mathbf{X}}^{\prime}(\mathbf{X})}{\sigma_{P}^{2}(\mathbf{X})} \frac{\partial g_{0}(\mathbf{X})}{\partial \mathbf{X}}\right)+O\left(\epsilon^{2}\right)
$$

where the function $g_{0}(\mathbf{X})$ is

$$
g_{0}(\mathbf{X})=\ln \rho-1+\mathrm{E}_{0}\left[\int_{0}^{\infty} e^{-\rho t}\left(r\left(\mathbf{X}_{t}\right)+\frac{\phi\left(\mathbf{X}_{t}\right)^{2} \sigma_{P}\left(\mathbf{X}_{t}\right)^{2}}{2}\right) d t \mid \mathbf{X}_{0}=\mathbf{X}\right]
$$

\subsection{Consumption and portfolio policies in the presence of portfolio constraints}

Up to this point, it had been assumed that the agent's consumption-portfolio choice was unconstrained. We now extend the analysis to allow for constraints on the portfolio weights. To simplify 
the exposition, we analyze only the infinite-horizon problem explicitly. It should be clear from our presentation how the solution of the finite-horizon problem in Proposition 2.1 must be modified to account for constraints.

We consider constraints of the form that restrict the portfolio weight on the risky asset to lie between a lower and an upper bound:

$$
\underline{\pi}(\mathbf{X}) \leq \pi(\mathbf{X}) \leq \bar{\pi}(\mathbf{X})
$$

where these bounds are allowed to depend on the state of the economy. Other than allowing for state-dependence, this specification of portfolio constraints is a specialization of the formulation in Cvitanic and Karatzas (1992) to the case of one risky asset. By restricting our attention to the constraint on portfolio proportions, we are ruling out more general types of constraints, e.g., the constraints on the absolute amount invested in each asset (see Grossman and Vila (1992), Cuoco (1997)).

The value function of the agent's constrained optimization problem now satisfies

$$
0=\max _{c, \pi \in[\pi(\mathbf{X}), \bar{\pi}(\mathbf{X})]}\left\{\begin{array}{c}
\frac{1}{\epsilon a}\left((W c)^{\epsilon a}-1\right)-\rho J+\left(r+\pi \phi \sigma_{P}^{2}-c\right) J_{W} W+\frac{1}{2} \pi^{2} W^{2} J_{W W} \sigma_{P}^{2} \\
+\mu_{\mathbf{X}}^{\prime} \cdot J_{\mathbf{X}}+\frac{1}{2} \sigma_{\mathbf{X}}^{\prime} \cdot J_{X X} \cdot \sigma_{\mathbf{X}}+\pi W \sigma_{P \mathbf{X}}^{\prime} \cdot J_{W \mathbf{X}}
\end{array}\right\}
$$

Proposition 2.3 In the presence of constraints, the optimal portfolio choice is given by

$$
\pi(\mathbf{X})= \begin{cases}\tilde{\pi}(\mathbf{X}), & \underline{\pi}(\mathbf{X}) \leq \tilde{\pi}(\mathbf{X}) \leq \bar{\pi}(\mathbf{X}) \\ \underline{\pi}(\mathbf{X}), & \tilde{\pi}(\mathbf{X})<\underline{\pi}(\mathbf{X}) \\ \bar{\pi}(\mathbf{X}), & \tilde{\pi}(\mathbf{X})>\bar{\pi}(\mathbf{X})\end{cases}
$$

where

$$
\widetilde{\pi} \equiv \phi(\mathbf{X})+\epsilon a\left(\phi(\mathbf{X})+\frac{1}{\sigma_{P}^{2}(\mathbf{X})} \sigma_{P \mathbf{X}}^{\prime}(\mathbf{X}) \cdot \frac{\partial g_{0}^{\mathrm{c}}(\mathbf{X})}{\partial \mathbf{X}}\right)+O\left(\epsilon^{2}\right)
$$

and the optimal consumption policy is given by

$$
c(\mathbf{X})=\rho-\epsilon a \rho\left(g_{0}^{\mathrm{c}}(\mathbf{X})-\ln (\rho)\right)+O\left(\epsilon^{2}\right) .
$$

The value function of the log investor in the presence of constraints is

$$
\begin{aligned}
g_{0}^{\mathrm{c}}(\mathbf{X})= & \ln \rho-1 \\
& +\mathrm{E}\left[\int_{0}^{\infty} e^{-\rho t}\left(r\left(\mathbf{X}_{t}\right)+\pi_{0}\left(\mathbf{X}_{t}\right) \phi\left(\mathbf{X}_{t}\right) \sigma_{P}\left(\mathbf{X}_{t}\right)^{2}-\frac{1}{2} \pi_{0}^{2}\left(\mathbf{X}_{t}\right) \sigma_{P}^{2}\left(\mathbf{X}_{t}\right)\right) d t \mid \mathbf{X}_{0}=\mathbf{X}\right]
\end{aligned}
$$


where

$$
\pi_{0}\left(\mathbf{X}_{t}\right)= \begin{cases}\phi\left(\mathbf{X}_{t}\right), & \underline{\pi}\left(\mathbf{X}_{t}\right) \leq \phi\left(\mathbf{X}_{t}\right) \leq \bar{\pi}\left(\mathbf{X}_{t}\right), \\ \underline{\pi}\left(\mathbf{X}_{t}\right), & \phi\left(\mathbf{X}_{t}\right)<\underline{\pi}\left(\mathbf{X}_{t}\right), \\ \bar{\pi}\left(\mathbf{X}_{t}\right), & \phi\left(\mathbf{X}_{t}\right)>\bar{\pi}\left(\mathbf{X}_{t}\right) .\end{cases}
$$

The function $g_{0}^{\mathrm{c}}(\mathbf{X})$ in (14), where the superscript "c" indicates the presence of constraints, is the counterpart of the function $g_{0}(\mathbf{X})$ in (13): it defines the value function of the log-utility maximizer subject to the same portfolio constraints and the same investment opportunity set as the investor with (non-log) power utility function.

As in the unconstrained case, an explicit asymptotic expression for the optimal consumption and portfolio policies is available as long as the solution of the analogous problem for the agent with the logarithmic utility function is known in closed form.

\subsection{Consumption and portfolio policies in general equilibrium}

The results in Propositions 2.1, 2.2 and 2.3 can be used as building blocks in the analysis of a broad range of models. In particular, they allow one to obtain asymptotic expressions for the prices of assets in equilibrium economies that otherwise can only be studied numerically. Successful application of our results is possible as long as it is possible to obtain explicit solutions for agents with logarithmic utility functions. In that case, the asymptotic demand functions, equations (7) and (8) for the finite-horizon case or (12) and (13) for the infinite-horizon case, are known in closed form and for equilibrium models the asset prices can be determined from the market clearing conditions.

We now explain how the results of the previous section can be used to analyze heterogeneousagent economies, and in the next section we study a particular application. Specifically, consider an infinite-horizon economy populated by heterogeneous agents, each with power utility function. Recall that $\gamma \equiv \epsilon a$, where differences in $a$ lead to differences in risk aversion, while $\epsilon$ controls the magnitude of these differences. In such an economy the investment opportunity set depends on the cross-sectional distribution of wealth among the agents, as well as on the exogenous state variables and the small parameter $\epsilon$. Thus, the expanded state vector is given by

$$
\mathbf{X}_{t}=\left\{\mathbf{X}_{0 t},\left\{W_{t}(a)\right\}\right\}
$$


where $\mathbf{X}_{0 t}$ denotes the vector of exogenous state variables and $\left\{W_{t}(a)\right\}$ is the set of individual wealth values for the agents in the economy.

Because the moments of returns on financial assets explicitly depend on $\epsilon$, we can approximate them by an asymptotic power series. Specifically, a moment of returns $m_{t}$ can be expressed as

$$
m_{t}=m\left(\mathbf{X}_{t}, \epsilon\right)=m_{0}\left(\mathbf{X}_{0 t}\right)+\epsilon m_{1}\left(\mathbf{X}_{t}\right)+O\left(\epsilon^{2}\right)
$$

The leading term in the expansion coincides with the corresponding moment in an economy with $\epsilon=0$, i.e., in a homogeneous-agent economy with log-utility maximizing agents. This implies that $m_{0}$ depends only on the exogenous state vector $\mathbf{X}_{0 t}$.

Next, consider the term $g_{0}(\mathbf{X}, \epsilon)$ in the value function of the log-utility maximizer in our heterogeneous-agent economy. Since the moments of returns depend on $\epsilon$, so does the function $g_{0}$, according to Proposition 2.2. Thus,

$$
g_{0}(\mathbf{X}, \epsilon)=g_{0,0}\left(\mathbf{X}_{0}\right)+\epsilon g_{0,1}(\mathbf{X})+O\left(\epsilon^{2}\right) .
$$

The leading term $g_{0,0}$ corresponds to the value function in the homogeneous-agent economy with log-utility maximizing agents and hence does not depend on the wealth distribution.

We can now use Proposition 2.2 to obtain asymptotic expansions for the individual portfolio policies in a heterogeneous-agent economy.

Proposition 2.4 The optimal consumption and portfolio policies in a heterogenous-agent economy are given by:

$$
\begin{aligned}
& c(\mathbf{X})=\rho-\epsilon a \rho\left(g_{0,0}\left(\mathbf{X}_{0}\right)-\ln \rho\right)+O\left(\epsilon^{2}\right) \\
& \pi(\mathbf{X})=\phi(\mathbf{X})+\epsilon a\left(\phi(\mathbf{X})+\frac{\sigma_{P \mathbf{X}, 0}^{\prime}\left(\mathbf{X}_{0}\right)}{\sigma_{P, 0}^{2}\left(\mathbf{X}_{0}\right)} \frac{\partial g_{0,0}\left(\mathbf{X}_{0}\right)}{\partial \mathbf{X}_{0}}\right)+O\left(\epsilon^{2}\right)
\end{aligned}
$$

where $g_{0,0}$ is given in (18).

Thus, to obtain a valid asymptotic expansion of the consumption and portfolio policies in a general equilibrium economy one simply has to replace the function $g_{0}$ in Proposition 2.2 with it's leading term $g_{0,0}$. This greatly simplifies computation of the equilibrium, because the function $g_{0,0}$ 
corresponds to the value function of the log investor in a homogeneous economy, and therefore, can be obtained without solving for the equilibrium in the heterogeneous economy. In the next section, we show how one can use the method developed above to analyze a heterogenous-agent economy in the presence of portfolio constraints.

\section{A general equilibrium exchange economy}

In this section, we study an exchange (endowment) economy with multiple agents who differ in their level of risk aversion. ${ }^{5}$ Wang (1996) analyzes this economy for the case where there are two agents who do not face any portfolio constraints. ${ }^{6}$ We extend the analysis of Wang in several directions. First, we show how one can characterize the equilibrium for the case where there is an arbitrary number of agents. In contrast to Wang, we also obtain closed-form (asymptotic) expressions for the mean and volatility of the stock return process. This analysis allows us to relate the volatility of stock returns to the heterogeneity of investors in their degree of risk aversion and to the cross-sectional dispersion in stock holdings.

Second, in Section 3.2, we introduce a leverage constraint that restricts how much investors can borrow to lever their investment in the stock. The model can no longer be solved using the representative-agent approach used in Wang (1996). We use the asymptotic approach to characterize the equilibrium in the presence of constraints, first for the case where there are only two agents, and then for the case where there is a continuum of agents. We then analyze the relation between the constraint on leverage and the volatility of stock returns, and also the interaction between the leverage constraint and heterogeneity across investors.

\subsection{The economy with unconstrained agents}

We assume that there are two assets available for trading in the economy. The first asset is a short-term risk-free bond, available in zero net supply, which pays the interest rate $r_{t}$ that will be determined in equilibrium. The second asset is a stock, which is a claim on the aggregate

\footnotetext{
${ }^{5}$ We report only the analysis of the exchange economy considered in Wang (1996); the analysis of the production economy studied in Dumas (1989), with the addition of portfolio constraints, can be obtained from the authors.

${ }^{6}$ Wang (1996) also discusses how the model could be solved when there are up to 4 agents, each having a particular value for the risk aversion parameter; with more than 4 agents a closed-form solution is not available for general wealth distributions.
} 
endowment, $e_{t}$, that evolves according to

$$
d e_{t}=\mu_{e} e_{t} d t+\sigma_{e} e_{t} d Z_{e t},
$$

where $\mu_{e}$ and $\sigma_{e}$ are constant parameters. We assume that the growth rate of the endowment is positive, $\mu_{e}-\sigma_{e}^{2} / 2>0$. The cumulative stock return process is

$$
\frac{d P_{t}+e_{t} d t}{P_{t}}=\mu_{R t} d t+\sigma_{R t} d Z_{t}
$$

with $\mu_{R t}$ and $\sigma_{R t}$ to be determined in equilibrium.

Assume that agents in the economy differ in their degree of risk aversion, $\gamma(a)=\epsilon a$. Without loss of generality, assume that there is a single agent of each type $a$. Let $W_{t}(a)$ be the wealth of the individual agent, $\sum_{a} W_{t}(a)$ is the aggregate wealth in the economy, which is also equal to the value of the stock market, $P_{t}$, and $\omega_{t}(a) \equiv W_{t}(a) / \sum_{a} W_{t}(a)$. The investment opportunity set depends on the cross-sectional distribution of wealth across agents, and thus, the correspondence with the general formulation in Section 2 is that $\mathbf{X}_{t}=\left\{W_{t}(a)\right\}$. If $\epsilon$ were equal to zero, then all the agents would have logarithmic preferences. As a result, they would hold the same portfolio and their wealth would be perfectly correlated; in this case, the cross-sectional distribution of wealth in the economy would be constant over time.

\subsubsection{Individual policies}

We start by identifying the value function of an agent in an economy where $\epsilon=0$ (that is, all agents have $\log$ utility). In such an economy, the value function of a representative agent equals

$$
\frac{1}{\rho}\left(\ln W_{0}+g_{0,0}\right)=\mathrm{E}_{0}\left[\int_{0}^{\infty} e^{-\rho t} \ln e_{t} d t\right]
$$

The expectation on the right-hand side equals $\frac{1}{\rho} \ln e_{0}+A$, where

$$
A \equiv \frac{1}{\rho}\left(\mu_{e}-\frac{\sigma_{e}^{2}}{2}\right)
$$

and also, $W_{0}$ is the aggregate wealth in the economy, which is equal to the price of the stock, $\frac{1}{\rho} e_{0}$. Thus,

$$
g_{0,0}=\ln \rho+A .
$$


Substituting the above value for $g_{0,0}$ into Proposition 2.4 then leads to the consumption and portfolio policies:

$$
\begin{aligned}
& c_{t}(a)=\rho-\epsilon a \rho A+O\left(\epsilon^{2}\right) \\
& \pi_{t}(a)=(1+\epsilon a) \phi_{t}+O\left(\epsilon^{2}\right),
\end{aligned}
$$

where the market price of risk, $\phi_{t}=\left(\mu_{R t}-r_{t}\right) / \sigma_{R t}^{2}$, along with the riskless interest rate, $r_{t}$, is determined in equilibrium.

\subsubsection{Equilibrium}

The equilibrium in this economy is defined by the stock price process, $P_{t}$, the interest rate process $r_{t}$, and the portfolio and consumption policies, such that (i) given the price processes for financial assets, the consumption and portfolio choices are optimal for the agents, (ii) the goods market and the markets for the stock and the bond clear.

The conditions for market clearing in the stock and commodity markets are:

$$
\begin{aligned}
\sum_{a} \pi_{t}(a) \omega_{t}(a) & =1 \\
\sum_{a} c_{t}(a) W_{t}(a) & =e_{t}
\end{aligned}
$$

where, if one wishes to consider a continuum of agents, the summation signs should be replaced by integrals. Using these market-clearing conditions along with the expressions for the optimal consumption and portfolio polices for individual investors, and defining

$$
\mathrm{E}_{a}[a] \equiv \sum_{a} a \omega_{t}(a), \quad \operatorname{var}_{a}[a] \equiv \mathrm{E}_{a}\left[a^{2}\right]-\left(\mathrm{E}_{a}[a]\right)^{2}
$$

we have the following characterization of the equilibrium in the unconstrained economy.

Proposition 3.1 For the exchange economy described above, in equilibrium:

(i) The stock price is given by

$$
\frac{P_{t}}{e_{t}}=\frac{1}{\rho}+\epsilon \frac{1}{\rho} A \mathrm{E}_{a}[a]+O\left(\epsilon^{2}\right)
$$

while the moments of the cumulative return process are 


$$
\begin{aligned}
\mu_{R t} & =\left(\mu_{e}+\rho\right)-\epsilon \rho A \mathrm{E}_{a}[a]+O\left(\epsilon^{2}\right), \\
\sigma_{R t} & =\sigma_{e}+\epsilon^{2} A \sigma_{e} \operatorname{var}_{a}[a]+O\left(\epsilon^{3}\right)
\end{aligned}
$$

(ii) The interest rate is given by

$$
r_{t}=\left(\mu_{e}-\sigma_{e}^{2}+\rho\right)+\epsilon\left(\sigma_{e}^{2}-\rho A\right) \mathrm{E}_{a}[a]+O\left(\epsilon^{2}\right) .
$$

(iii) The optimal portfolio policy is

$$
\pi_{t}(a)=1+\epsilon\left(a-\mathrm{E}_{a}[a]\right)+O\left(\epsilon^{2}\right) .
$$

(iv) The cross-sectional wealth distribution evolves according to

$$
\frac{d \omega_{t}(a)}{\omega_{t}(a)}=\epsilon \rho A\left(a-\mathrm{E}_{a}[a]\right) d t+\epsilon \sigma_{e}\left(a-\mathrm{E}_{a}[a]\right) d Z_{t}+O\left(\epsilon^{2}\right)
$$

Observe that the first moment of stock returns in (25) can be computed only up to order $O\left(\epsilon^{2}\right)$ terms because we know $P_{t} / e_{t}$ only up to $O\left(\epsilon^{2}\right)$ terms. However, the second moment, given in equation (26), is known to higher order. Based on this, we have the following result.

Proposition 3.2 Asymptotically, the volatility of stock returns is increasing in the cross-sectional heterogeneity of risk aversion.

To understand the intuition behind this result, consider the equilibrium stock price (24). The price-dividend ratio is decreasing in average risk aversion. This is because the expected stock return is increasing in average risk aversion, as shown in (25). Moreover, the average risk aversion in the economy fluctuates over time in response to the aggregate endowment shocks. According to (21), agents with relatively high risk aversion are less exposed to the stock market risk. Therefore, the fraction of total wealth controlled by agents with higher-than-average risk aversion declines as the stock market rises, as shown in (29). As a result, the average risk aversion in the economy is negatively affected by the aggregate endowment shocks, implying a positive effect on the pricedividend ratio. ${ }^{7}$ The positive impact of the endowment shocks on the price-dividend ratio increases

\footnotetext{
${ }^{7}$ Chan and Kogan (2001) discuss the countercyclical nature of expected stock returns due to investor heterogeneity in a setting were individuals have catching-up-with-the-Joneses preferences. Our setting uses a more common specification of individual preferences, but the same intuition for time-variation in expected returns applies in both cases. While Chan and Kogan rely on numerical analysis and focus on the dynamics of conditional moments of stock returns, we derive an explicit asymptotic relation between the level of return volatility and the degree of cross-sectional heterogeneity.
} 
the volatility of stock returns. Because the outlined effect is due to the cross-sectional differences in investors' risk aversion, it comes as no surprise that its magnitude is related to the cross-sectional dispersion of individual types, as captured by (26).

Given individual risk aversion coefficients are not directly observable, it is useful to re-state Proposition 3.2 in terms of individual portfolio choices. Because $\operatorname{var}_{a}\left[\pi_{t}(a)\right]=\epsilon^{2} \operatorname{var}_{a}[a]+O\left(\epsilon^{3}\right)$, there exists a positive linear asymptotic relation between the volatility of stock returns and the cross-sectional dispersion of individual portfolio holdings.

\subsubsection{Comparison with the exact solution}

In order to demonstrate that the analytical results described above capture the salient qualitative features of the exact solution, we solve numerically the unconstrained model for the case where there are only two agents. Based on the exact solution obtained numerically, we present in Figure 1 the parametric plots showing the relation between the cross-sectional dispersion of portfolio holdings $\left(\operatorname{var}_{a}[\pi(a)]\right)$ and the conditional volatility of stock returns (scaled by the volatility of endowment process). There are four plots, each for a different degree of heterogeneity across the two investors, which is given by the parameter $\epsilon$. The solid line in each plot corresponds to the asymptotic solution while the dashed line is for the exact numerical solution.

Consistent with our analytic asymptotic results, all four plots show that the volatility of stock returns tends to increase with the cross-sectional dispersion of portfolio holdings. Given the nature of the asymptotic expansions we are using, it is not surprising to find that the asymptotic solution is closer to the exact solution in the first three plots, where the degree of heterogeneity in the economy is smaller ( $\epsilon$ closer to zero) and investors have risk aversion closer to unity, relative to the fourth plot, where $\epsilon$ is much further away from zero. Nevertheless, even in the latter case the relation between return volatility and cross-sectional dispersion is positive.

\subsection{The economy with portfolio constraints}

Now, assume that agents are restricted in the amount they can borrow in order to lever up their investment in the stock. In particular, individual portfolio positions must satisfy:

$$
\pi_{t} \leq 1+\epsilon L
$$


Observe that we specify the limit on borrowing, $L$, to be proportional to the small parameter $\epsilon$. This is because the equilibrium portfolio policies in the unconstrained economy, as given by Proposition 3.1, imply that the amount of borrowing by individual agents is proportional to $\epsilon$; therefore, in order for the leverage constraint to have an impact for small values of the small parameter, it must be sufficiently tight, i.e., formally, it must be proportional to $\epsilon$ as well.

In the presence of the leverage constraint, based on Proposition 2.3 the individual portfolio policies take the form

$$
\begin{aligned}
\pi_{t}(a) & =\min \left[(1+\epsilon a) \phi_{t}, 1+\epsilon L\right]+O\left(\epsilon^{2}\right) \\
& =1+\epsilon \min \left[a+\phi_{1 t}, L\right]+O\left(\epsilon^{2}\right),
\end{aligned}
$$

where the market price of risk is expanded as $\phi_{t}=1+\epsilon \phi_{1 t}+O\left(\epsilon^{2}\right) .{ }^{8}$ We will say that the leverage constraint is binding for an agent of type $a$ when $b+\phi_{1 t} \geq L$.

\subsubsection{Equilibrium in the constrained economy with two agents}

We start by considering the case where there are only two classes of agents, $a^{\prime}$ and $a^{\prime \prime}$, with $a^{\prime}<a^{\prime \prime}$. Then, the market clearing condition in the stock market is:

$$
\begin{aligned}
1 & =\sum_{a} \pi_{t}(a) \omega_{t}(a) \\
& =1+\epsilon \sum_{a} \min \left[a+\phi_{1 t}, L\right] \omega_{t}(a)+O\left(\epsilon^{2}\right)
\end{aligned}
$$

Proceeding as before, by first identifying $g_{0,0}^{\mathrm{c}}$, then identifying the optimal consumption and portfolio policies, and finally using the market clearing conditions to obtain the price processes, we have the following.

Proposition 3.3 In equilibrium, the leverage constraint is binding when $\omega_{t}\left(a^{\prime}\right)\left(a^{\prime \prime}-a^{\prime}\right)-L \geq 0$. In this region:

\footnotetext{
agents.

${ }^{8}$ The leading term in the expansion $\phi_{0 t}=1$ is the market price of risk in the economy populated with log-utility
} 
(i) The stock price is given by

$$
\left.\frac{P_{t}}{e_{t}}=\frac{1}{\rho}+\epsilon \frac{1}{\rho} A \mathrm{E}_{a}[a]\right)+O\left(\epsilon^{2}\right)
$$

while the moments of the cumulative return process are

$$
\begin{aligned}
\mu_{R t} & =\left(\mu_{e}+\rho\right)-\epsilon \rho A \mathrm{E}_{a}[a]+O\left(\epsilon^{2}\right), \\
\sigma_{R t} & =\sigma_{e}+\epsilon^{2} A \sigma_{e} L\left[a^{\prime \prime}-a^{\prime}\right] \omega_{t}\left(a^{\prime \prime}\right)+O\left(\epsilon^{3}\right) .
\end{aligned}
$$

(ii) The interest rate is

$$
r_{t}=\left(\mu_{e}-\sigma_{e}^{2}+\rho\right)+\epsilon\left(\sigma_{e}^{2}\left(L \frac{\omega_{t}\left(a^{\prime \prime}\right)}{\omega_{t}\left(a^{\prime}\right)}+a^{\prime}\right)-\rho A \mathrm{E}_{a}[a]\right)+O\left(\epsilon^{2}\right)
$$

(iii) The portfolio policy is

$$
\pi_{t}(a)=1+\epsilon \min \left[a-L \frac{\omega_{t}\left(a^{\prime \prime}\right)}{\omega_{t}\left(a^{\prime}\right)}-a^{\prime}, L\right]+O\left(\epsilon^{2}\right)
$$

(iv) The cross-sectional wealth distribution for the two types of agents evolves according to:

$$
\begin{aligned}
& \frac{d \omega_{t}\left(a^{\prime}\right)}{\omega_{t}\left(a^{\prime}\right)}=\epsilon \rho A\left(a^{\prime}-\mathrm{E}_{a}[a]\right) d t-\epsilon \sigma_{e} L \frac{\omega_{t}\left(a^{\prime \prime}\right)}{\omega_{t}\left(a^{\prime}\right)} d Z_{t}+O\left(\epsilon^{2}\right), \\
& \frac{d \omega_{t}\left(a^{\prime \prime}\right)}{\omega_{t}\left(a^{\prime \prime}\right)}=\epsilon \rho A\left(a^{\prime \prime}-\mathrm{E}_{a}[a]\right) d t+\epsilon \sigma_{e} L d Z_{t}+O\left(\epsilon^{2}\right) .
\end{aligned}
$$

When the leverage constraint is not binding, the solution is asymptotically the same as in the unconstrained case.

Imposing the leverage constraint lowers the risk free interest rate. Formally, the difference between interest rates in the unconstrained and the constrained economies, given by

$$
\epsilon \sigma_{e}^{2} \frac{\omega_{t}\left(a^{\prime \prime}\right)}{\omega_{t}\left(a^{\prime}\right)}\left(\omega_{t}\left(a^{\prime}\right)\left(a^{\prime \prime}-a^{\prime}\right)-L\right)+O\left(\epsilon^{2}\right)
$$

is asymptotically positive, because $\omega_{t}\left(a^{\prime}\right)\left(a^{\prime \prime}-a^{\prime}\right)-L \geq 0$ whenever the leverage constraint is binding. The expected stock return is asymptotically unaffected, according to (30). Thus, tightening the leverage constraint increases the equilibrium equity premium, $\mu_{R t}-r_{t} .{ }^{9}$

\footnotetext{
${ }^{9}$ Heaton and Lucas (1996) observe similar behavior of asset returns in their incomplete-market model in response to an increased difference in borrowing and lending rates. Their analysis relies on numerical simulations and the intuition behind their results is different. In their model, individuals have the same risk aversion but face idiosyncratic endowment shocks. As a result, an increase in trading costs raises individual consumption variability, and hence, lowers the risk-free rate of return due to the demand for precautionary savings.
} 
We now explain the intuition for this result. Start by considering the situation where trading in the stock is not allowed in equilibrium. Then, one class of agents would borrow to increase their current consumption, thereby reducing the growth rate of their consumption. Hence, imposing the borrowing constraint would reduce current consumption and increase the consumption growth rate of the constrained agent while reducing the growth rate for the unconstrained agent. This would lower the equilibrium interest rate, which is linked to the consumption growth rate of the unconstrained agent.

Now, consider the situation where agents can trade also the risky asset. This complicates matters in general. Intuitively, holding the asset price processes fixed, adding the leverage constraint reduces the aggregate demand for the risk free asset, which suggests that tightening the leverage constraint would result in a lower interest rate in equilibrium. However, this argument ignores the potential impact of the constraint on stock returns. We find that the leverage constraint has only a higher-order effect on the moments of stock returns in our model, as can be seen from comparing Propositions 3.1 and 3.3. To understand the reason for this, note that the stock price is determined by market clearing for the consumption good, and the consumption policy (the consumption to wealth ratio) itself is affected by the leverage constraint only through its effect on the investment opportunity set. Because the consumption policy of investors with logarithmic preferences is independent of the investment opportunity set, for agents with utility functions close to logarithmic, the impact of changes in the investment opportunity set on the consumption policy is of order $\epsilon$ (see Proposition 2.3). Moreover, the time-varying component of the investment opportunity set is itself of order $\epsilon$ in equilibrium, since the economy is perturbed around the logarithmic representative agent case where the investment opportunities are constant over time. Therefore, these two effects imply that the impact of the leverage constraint on consumption policies is of order $\epsilon^{2}$ (see Proposition 2.4). Thus, the constraint has only a second-order effect on the equilibrium price-dividend ratio and the moments of stock returns.

The above argument explains the impact of the leverage constraint on the risk-free rate and the equity premium in our model. We summarize these observations in the following proposition.

Proposition 3.4 Asymptotically, the interest rate in the constrained economy is lowered and the equity premium is increased by tightening the borrowing constraint. 
Next, we relate the volatility of stock returns to the leverage constraint and compare it to the volatility in the unconstrained economy.

Proposition 3.5 Asymptotically, the volatility of stock returns in the constrained economy is lower than in the unconstrained economy:

$$
\sigma_{R t}^{\mathrm{c}} \leq \sigma_{R t}^{\mathrm{u}}+O\left(\epsilon^{3}\right)
$$

where $\sigma_{R t}^{\mathrm{c}}$ and $\sigma_{R t}^{\mathrm{u}}$ denote the volatility of stock returns in the constrained and the unconstrained economy respectively. Moreover, the volatility of stock returns is reduced by tightening the borrowing constraint.

As we argued above, the volatility of stock returns is positively related to the variability of the average risk aversion in the economy. The leverage constraint reduces the cross-sectional differences in individual portfolio holdings, and hence, the variability of the cross-sectional wealth distribution and the average risk aversion. As a result, the constraint on borrowing lowers the volatility of stock returns.

\subsubsection{The economy with portfolio constraints and a continuum of agents}

In the previous section, we found that constraints on leverage lower the volatility of stock returns. We carried out our analysis for the special case of only two types of agents. In this section, we extend this result to an economy with a continuum of agents.

Assume that agents have risk aversion between $-\hat{a}$ and $+\hat{a}$, with an arbitrary wealth distribution, $\omega_{t}(a)$. Then, market clearing in the stock market implies

$$
0=\int_{-\hat{a}}^{L-\phi_{1 t}}\left(a+\phi_{1 t}\right) \omega_{t}(a) d a+\int_{L-\phi_{1 t}}^{\hat{a}} L \omega_{t}(a) d a,
$$

and, the volatility of the returns process is:

$$
\begin{aligned}
d\left(\frac{P_{t}}{e_{t}}\right) & =\left(\epsilon \frac{A}{\rho}+O\left(\epsilon^{2}\right)\right) \int_{-\hat{a}}^{\hat{a}} a d \omega_{t}(a) d a \\
& =[\ldots] d t+\epsilon^{2} \frac{A \sigma_{e}}{\rho}\left[\int_{-\hat{a}}^{L-\phi_{1 t}} a \omega_{t}(a)\left(a+\phi_{1 t}\right) d a+\int_{L-\phi_{1 t}}^{\hat{a}} L a \omega_{t}(a) d a\right]+O\left(\epsilon^{3}\right),
\end{aligned}
$$


which then allows us to show the following.

Proposition 3.6 Asymptotically, the interest rate in the constrained economy is lowered, the equity premium is increased, and the volatility of stock returns is reduced by tightening the borrowing constraint.

\section{Conclusion}

In this article, we have provided an asymptotic analysis of the optimal consumption and portfolio decisions of an investor who has preferences over intermediate consumption and faces an economic environment with stochastic investment opportunities and portfolio constraints. Our results include comparative statics results for optimal policies and analytic asymptotic expressions for equilibrium asset prices. In addition to the analysis of the portfolio policy of a single agent, we have shown how the portfolio-choice problem in the presence of a stochastic investment opportunity set can be embedded in a general equilibrium setting, even when there are multiple investors who differ in their degree of risk aversion and face constraints on their portfolio positions. Throughout our analysis, we have not needed to rely on the assumption that financial markets are complete.

The model developed in the paper can be extended in several directions. For instance, we have assumed that agents have time-additive power utility rather than the more general recursive preferences described in Kreps and Porteus (1978) and Duffie and Epstein (1992). Given that log utility is a special case also of the Kreps-Porteus specification of recursive utility, it is possible to extend the asymptotic method to the case of recursive preferences. Similarly, the method can also be applied to an economy where agents exhibit habit-persistence. Moreover, the general equilibrium model studied in the paper is of an exchange economy but the method applies also to a production economy.

One limitation of the analysis we have presented is that it applies only to those situations where there exists a closed-form solution for an investor with logarithmic utility. However, even when an explicit solution does not exist for the log investor, one may apply asymptotic analysis, but with the perturbation now being around a parameter different from that governing risk aversion. 


\section{Appendix: Proofs for all propositions}

\section{Proof of Proposition 2.1}

The result follows by substituting (6) into (5). First- and higher-order terms in the expansion of $g(\mathbf{X})$ do not affect the first-order asymptotic expansion of the optimal consumption-portfolio policy. The equivalent asymptotic expression (9) is obtained by expanding (8) in powers of $\epsilon$ and eliminating terms of order two and higher.

To obtain $g_{0}$, we use the definition of the value function of the log-utility maximizer, with optimal consumption $c\left(\mathbf{X}_{t}, t\right)=\psi / A(t)$ substituted in,

$$
J\left(W_{t}, \mathbf{X}_{t}, t\right)=\psi \mathrm{E}_{t}\left[\int_{t}^{T} e^{-\rho(s-t)} \ln \left(\frac{W_{s}}{A(s)}\right) d s\right]+(1-\psi) e^{-\rho(T-t)} \mathrm{E}_{t}\left[\ln W_{T}\right],
$$

where the wealth process $W_{t}$ evolves according to

$$
\frac{d W_{t}}{W_{t}}=\left(-\frac{\psi}{A(t)}+r\left(\mathbf{X}_{t}\right)+\pi\left(\mathbf{X}_{t}, t\right)\left(\mu_{P}\left(\mathbf{X}_{t}\right)-r\left(\mathbf{X}_{t}\right)\right)\right) d t+\pi\left(\mathbf{X}_{t}, t\right) \sigma_{P}\left(\mathbf{X}_{t}\right) d Z_{P t}
$$

Thus,

$$
\ln \left(W_{s}\right)=\ln \left(W_{t}\right)+\int_{t}^{s}-\frac{\psi}{A(u)}+r\left(\mathbf{X}_{u}\right)+\frac{\phi\left(\mathbf{X}_{u}\right)^{2} \sigma_{P}\left(\mathbf{X}_{u}\right)^{2}}{2} d u+\int_{t}^{s} \phi\left(\mathbf{X}_{u}\right) \sigma_{P}\left(\mathbf{X}_{u}\right) d Z_{P u}
$$

where we have used the expression for the optimal portfolio policy of the log investor, $\pi\left(\mathbf{X}_{t}, t\right)=$ $\phi\left(\mathbf{X}_{t}\right)$. Substituting this into (A1) yields

$$
\begin{aligned}
& J\left(W_{t}, \mathbf{X}_{t}, t\right)= \\
& \psi \mathrm{E}_{t}\left[\int_{t}^{T} e^{-\rho(s-t)}\left(\ln \psi-\ln A(s)+\left(\ln \left(W_{t}\right)+\int_{t}^{s} \frac{-\psi}{A(u)}+r\left(\mathbf{X}_{u}\right)+\frac{\phi\left(\mathbf{X}_{u}\right)^{2} \sigma_{P}\left(\mathbf{X}_{u}\right)^{2}}{2} d u\right)\right) d s\right] \\
& \quad+(1-\psi) e^{-\rho(T-t)} \mathrm{E}_{t}\left[\left(\ln \left(W_{t}\right)+\int_{t}^{T} \frac{-\psi}{A(u)}+r\left(\mathbf{X}_{u}\right)+\frac{\phi\left(\mathbf{X}_{u}\right)^{2} \sigma_{P}\left(\mathbf{X}_{u}\right)^{2}}{2} d u\right)\right] .
\end{aligned}
$$

Integration by parts completes the proof of the proposition.

\section{Proof of Proposition 2.2}

The results follow by setting $\psi=1$ and taking the limit as $T \rightarrow \infty$ for the corresponding expressions in Propositions 2.1 while noting that in the limit, $A(t)=1 / \rho$. 


\section{Proof of Proposition 2.3}

As in Proposition 2.2, this result follows by replacing the function $g(\mathbf{X})$ in the expression for the optimal consumption-portfolio policy with its asymptotic expansion. Only the leading term in the expansion must be retained, which corresponds to the solution of the log-utility maximizer's problem.

To obtain $g_{0}^{c}$ note that the wealth process of the log-investor evolves according

$$
\frac{d W_{t}}{W_{t}}=\left(-\rho+r\left(\mathbf{X}_{t}\right)+\pi_{0}\left(\mathbf{X}_{t}\right) \phi\left(\mathbf{X}_{t}\right) \sigma_{P}\left(\mathbf{X}_{t}\right)^{2}\right) d t+\pi_{0}\left(\mathbf{X}_{t}\right) \sigma_{P}\left(\mathbf{X}_{t}\right) d Z_{P t}
$$

where $\pi_{0}\left(\mathbf{X}_{t}\right)$ is the optimal portfolio policy of the log-utility maximizer, given by (16). Repeating the steps of the proof of Proposition 2.2 we obtain the desired result.

\section{Proof of Proposition 2.4}

Substituting the expansions (17) and (18) into the expression for the consumption policy and portfolio weights in Proposition 2.2, and eliminating the higher-order terms, yields the result.

\section{Proof of Proposition 3.1}

Divide equation (23) by aggregate wealth, which equals $P_{t}$, to get

$$
\frac{e_{t}}{P_{t}}=\sum_{a} c_{t}(a) \omega_{t}(a)
$$

According to Proposition 2.2, the individual consumption policy is

$$
c_{t}(a)=\rho-\epsilon a \rho A+O\left(\epsilon^{2}\right) .
$$

Together, these two results imply that

$$
\frac{P_{t}}{e_{t}}=\frac{1}{\rho} \frac{1}{\sum_{a}(1-\epsilon a A) \omega_{t}(a)}+O\left(\epsilon^{2}\right)=\frac{1}{\rho}\left(1+\epsilon A \mathrm{E}_{a}[a]\right)+O\left(\epsilon^{2}\right) .
$$

Using

$$
\frac{d P_{t}}{P_{t}}=\frac{d\left(P_{t} / e_{t}\right)}{\left(P_{t} / e_{t}\right)}+\frac{d e_{t}}{e_{t}}+\frac{d e_{t}}{e_{t}} \cdot \frac{d\left(P_{t} / e_{t}\right)}{P_{t} / e_{t}}
$$

and 


$$
d \omega_{t}(a)=O(\epsilon) d t+O(\epsilon) d Z_{t}
$$

we find that

$$
\frac{d P_{t}}{P_{t}}=\mu_{e} d t+\sigma_{e} d Z_{t}+O\left(\epsilon^{2}\right)
$$

which then implies that

$$
\begin{aligned}
\mu_{R t} & =\mu_{e}+\frac{e_{t}}{P_{t}}+O\left(\epsilon^{2}\right) \\
& =\left(\mu_{e}+\rho\right)-\epsilon \rho A \mathrm{E}_{a}[a]+O\left(\epsilon^{2}\right)
\end{aligned}
$$

and

$$
\sigma_{R t}=\sigma_{e}+O\left(\epsilon^{2}\right)
$$

Using the condition in equation (22) for equilibrium in the stock market,

$$
\frac{\mu_{R t}-r_{t}}{\sigma_{R t}^{2}}\left(1+\epsilon \mathrm{E}_{a}[a]\right)=1
$$

we have that

$$
\frac{\mu_{R t}-r_{t}}{\sigma_{R t}^{2}}=1-\epsilon \mathrm{E}_{a}[a]+O\left(\epsilon^{2}\right)
$$

so that

$$
\begin{aligned}
r_{t} & =\mu_{R t}-\sigma_{R t}^{2}\left(1-\epsilon \mathrm{E}_{a}[a]\right)+O\left(\epsilon^{2}\right) \\
& =\left(\mu_{e}-\sigma_{e}^{2}+\rho\right)+\epsilon\left(\sigma_{e}^{2}-\rho A\right) \mathrm{E}_{a}[a]+O\left(\epsilon^{2}\right)
\end{aligned}
$$

Also, using (A5), the expression for the optimal portfolio weight in equation (21) reduces to

$$
\pi_{t}(a)=1+\epsilon\left(a-\mathrm{E}_{a}[a]\right)+O\left(\epsilon^{2}\right) .
$$

To derive the process for stock returns in terms of exogenous variables, and to determine the higher-order terms in the asymptotic expansions of the moments of the return process, we start by describing the evolution of $W_{t}(a)$ :

$$
\begin{aligned}
\frac{d W_{t}(a)}{W_{t}(a)}= & {\left[\pi_{t}(a)\left(\mu_{R t}-r_{t}\right)+r_{t}-c_{t}(a)\right] d t+\pi_{t}(a) \sigma_{R t} d Z_{t} } \\
= & {\left[\sigma_{e}^{2}\left(1-\epsilon \mathrm{E}_{a}[a]\right)\left(1+\epsilon\left(a-\mathrm{E}_{a}[a]\right)\right)+\mu_{e}-\sigma_{e}^{2}+\rho+\epsilon\left(\sigma_{e}^{2}-\rho A\right) \mathrm{E}_{a}[a]-\rho(1-\epsilon a A)\right] d t } \\
& +\sigma_{e}\left(1+\epsilon\left(a-\mathrm{E}_{a}[a]\right)\right) d Z_{t}+O\left(\epsilon^{2}\right)
\end{aligned}
$$




$$
\begin{aligned}
= & {\left[\sigma_{e}^{2}+\epsilon \sigma_{e}^{2}\left(a-2 \mathrm{E}_{a}[a]\right)+\mu_{e}-\sigma_{e}^{2}+\rho+\epsilon\left(\sigma_{e}^{2}-\rho A\right) \mathrm{E}_{a}[a]-\rho+\epsilon \rho A a\right] d t } \\
& +\sigma_{e}\left(1+\epsilon\left(a-\mathrm{E}_{a}[a]\right)\right) d Z_{t}+O\left(\epsilon^{2}\right) \\
= & {\left[\mu_{e}+\epsilon\left(\sigma_{e}^{2}+\rho A\right)\left(a-\mathrm{E}_{a}[a]\right)\right] d t+\sigma_{e}\left(1+\epsilon\left(a-\mathrm{E}_{a}[a]\right)\right) d Z_{t}+O\left(\epsilon^{2}\right) . }
\end{aligned}
$$

Next,

$$
\begin{aligned}
\frac{d \omega_{t}(a)}{\omega_{t}(a)}= & \frac{d W_{t}(a)}{W_{t}(a)}-\frac{d P_{t}}{P_{t}}+\frac{1}{P_{t}^{2}}\left[d P_{t}, d P_{t}\right]-\left[\frac{d W_{t}(a)}{W_{t}(a)}, \frac{d P_{t}}{P_{t}}\right] \\
= & {\left[\mu_{e}+\epsilon\left(\sigma_{e}^{2}+\rho A\right)\left(a-\mathrm{E}_{a}[a]\right)\right] d t+\sigma_{e}\left(1+\epsilon\left(a-\mathrm{E}_{a}[a]\right)\right) d Z_{t}-\mu_{e} d t-\sigma_{e} d Z_{t} } \\
& +\sigma_{e}^{2} d t-\sigma_{e}^{2}\left(1+\epsilon\left(a-\mathrm{E}_{a}[a]\right)\right) d t+O\left(\epsilon^{2}\right) \\
= & \epsilon \rho A\left(a-\mathrm{E}_{a}[a]\right) d t+\epsilon \sigma_{e}\left(a-\mathrm{E}_{a}[a]\right) d Z_{t}+O\left(\epsilon^{2}\right) .
\end{aligned}
$$

Finally, this leads to the following result: from (A2),

$$
\begin{aligned}
d\left(P_{t} / e_{t}\right) & =\left(\frac{\epsilon A}{\rho}\right)\left(\sum_{a} a d \omega_{t}(a)\right)+O\left(\epsilon^{2}\right) \cdot d \omega_{t}(a) \\
& =\epsilon^{2}\left(A^{2} \sum_{a} a\left(a-\mathrm{E}_{a}[a]\right) \omega_{t}(a)\right) d t+\epsilon^{2} \frac{A \sigma_{e}}{\rho} \sum_{a} a\left(a-\mathrm{E}_{a}[a]\right) \omega_{t}(a) d Z_{t}+O\left(\epsilon^{3}\right) \\
& =\epsilon^{2} A^{2} \operatorname{var}_{a}[a] d t+\epsilon^{2} \frac{A \sigma_{e}}{\rho} \operatorname{var}_{a}[a] d Z_{t}+O\left(\epsilon^{3}\right)
\end{aligned}
$$

and so, from (A3),

$$
\begin{aligned}
\frac{d P_{t}}{P_{t}} & =\epsilon^{2} \rho A^{2} \operatorname{var}_{a}[a] d t+\epsilon^{2} A \sigma_{e} \operatorname{var}_{a}[a] d Z_{t}+\mu_{e} d t+\sigma_{e} d Z_{t}+\epsilon^{2} A \sigma_{e}^{2} \operatorname{var}_{a}[a] d t+O\left(\epsilon^{3}\right) \\
& =\left(\mu_{e}+\epsilon^{2}\left(\rho A^{2}+\sigma_{e}^{2} A\right) \operatorname{var}_{a}[a]\right) d t+\left(\sigma_{e}+\epsilon^{2} A \sigma_{e} \operatorname{var}_{a}[a]\right) d Z_{t}+O\left(\epsilon^{3}\right) .
\end{aligned}
$$

\section{Proof of Proposition 3.2}

The result follows from differentiating the expression for the volatility of stock returns in (26) with respect to $\operatorname{var}_{a}[a]$.

\section{Proof of Proposition 3.3}

The introduction of portfolio constraints changes equation (27) and onwards, but because equations (24) and (25) are based on (20), these results are still valid. 
From equation (22):

$$
\begin{aligned}
1 & =\mathrm{E}_{a}\left[\pi_{t}(a)\right] \\
& =\mathrm{E}_{a}\left[(1+\epsilon a) \frac{\mu_{R t}-r_{t}}{\sigma_{R t}^{2}} \chi\left(a \leq L \frac{\sigma_{R t}^{2}}{\mu_{R t}-r_{t}}\right)\right]+\mathrm{E}_{a}\left[(1+\epsilon L) \chi\left(a>L \frac{\sigma_{R t}^{2}}{\mu_{R t}-r_{t}}\right)\right],
\end{aligned}
$$

where $\chi(\cdot)$ is an indicator function. This equation is used to determine $r$ as follows. Assuming that $a^{\prime}<a^{\prime \prime}$ and that the constraint binds for $a^{\prime \prime}$,

$$
1=\left(1+\epsilon a^{\prime}\right) \frac{\mu_{R t}-r_{t}}{\sigma_{R t}^{2}} \omega_{t}\left(a^{\prime}\right)+(1+\epsilon L) \omega_{t}\left(a^{\prime \prime}\right),
$$

which implies that

$$
\begin{aligned}
\frac{\mu_{R t}-r_{t}}{\sigma_{R t}^{2}} & =\frac{\omega_{t}\left(a^{\prime}\right)-\epsilon L \omega_{t}\left(a^{\prime \prime}\right)}{\omega_{t}\left(a^{\prime}\right)\left(1+\epsilon a^{\prime}\right)} \\
& =1-\epsilon\left(L \frac{\omega_{t}\left(a^{\prime \prime}\right)}{\omega_{t}\left(a^{\prime}\right)}+a^{\prime}\right)+O\left(\epsilon^{2}\right) \\
r_{t} & =\mu_{R t}-\sigma_{R t}^{2}\left[1-\epsilon\left(L \frac{\omega_{t}\left(a^{\prime \prime}\right)}{\omega_{t}\left(a^{\prime}\right)}+a^{\prime}\right)\right]+O\left(\epsilon^{2}\right) .
\end{aligned}
$$

Consider the region where the constraint is binding:

$$
\begin{aligned}
L & \leq a^{\prime \prime}-L \frac{\omega_{t}\left(a^{\prime \prime}\right)}{\omega_{t}\left(a^{\prime}\right)}-a^{\prime} \\
\Rightarrow \omega_{t}\left(a^{\prime}\right) & \geq \frac{L}{a^{\prime \prime}-a^{\prime}} .
\end{aligned}
$$

In this region,

$$
\begin{aligned}
\frac{d W_{t}\left(a^{\prime}\right)}{W_{t}\left(a^{\prime}\right)}= & {\left[\mu_{e}+\epsilon\left(\rho A\left(a^{\prime}-\mathrm{E}_{a}[a]\right)-\sigma_{e}^{2} L \frac{\omega_{t}\left(a^{\prime \prime}\right)}{\omega_{t}\left(a^{\prime}\right)}\right)\right] d t } \\
& +\sigma_{e}\left(1+\epsilon a^{\prime}\right)\left[1-\epsilon\left(L \frac{\omega_{t}\left(a^{\prime \prime}\right)}{\omega_{t}\left(a^{\prime}\right)}+a^{\prime}\right)\right] d Z_{t} \\
= & {\left[\mu_{e}+\epsilon\left(\rho A\left(a^{\prime}-\mathrm{E}_{a}[a]\right)+L \sigma_{e}^{2} L \frac{\omega_{t}\left(a^{\prime \prime}\right)}{\omega_{t}\left(a^{\prime}\right)}\right)\right] d t } \\
& +\sigma_{e}\left[1-\epsilon L \frac{\omega_{t}\left(a^{\prime \prime}\right)}{\omega_{t}\left(a^{\prime}\right)}\right] d Z_{t}+O\left(\epsilon^{2}\right) ; \\
\frac{d W_{t}\left(a^{\prime \prime}\right)}{W_{t}\left(a^{\prime \prime}\right)}= & {\left[\mu_{e}+\epsilon\left(\rho A\left(a^{\prime \prime}-\mathrm{E}_{a}[a]\right)+L\right)\right] d t+\sigma_{e}(1+\epsilon L) d Z_{t}+O\left(\epsilon^{2}\right) . }
\end{aligned}
$$


Thus,

$$
\begin{aligned}
\frac{d \omega_{t}\left(a^{\prime}\right)}{\omega_{t}\left(a^{\prime}\right)} & =\epsilon \rho A\left(a^{\prime}-\mathrm{E}_{a}[a]\right) d t-\epsilon \sigma_{e} L \frac{\omega_{t}\left(a^{\prime \prime}\right)}{\omega_{t}\left(a^{\prime}\right)} d Z_{t} \\
\frac{d \omega_{t}\left(a^{\prime \prime}\right)}{\omega_{t}\left(a^{\prime \prime}\right)} & =\epsilon \rho A\left(a^{\prime \prime}-\mathrm{E}_{a}[a]\right) d t+\epsilon \sigma_{e} L d Z_{t} .
\end{aligned}
$$

Using these results,

$$
\begin{aligned}
d\left(\frac{P_{t}}{e_{t}}\right) & =\left(\frac{\epsilon A}{\rho} d Z_{t}+O\left(\epsilon^{2}\right)\right)\left(\sum_{a} a d \omega_{t}(a)\right) \\
& =[\ldots] d t+\epsilon^{2} \frac{A \sigma_{e} L}{\rho}\left[a^{\prime \prime} \omega_{t}\left(a^{\prime \prime}\right)-a^{\prime} \omega_{t}\left(a^{\prime \prime}\right)\right] d Z_{t}+O\left(\epsilon^{3}\right) \\
& =[\ldots] d t+\epsilon^{2} \frac{A \sigma_{e} L}{\rho}\left[a^{\prime \prime}-a^{\prime}\right] \omega_{t}\left(a^{\prime \prime}\right) d Z_{t}+O\left(\epsilon^{3}\right) .
\end{aligned}
$$

So,

$$
\sigma_{R t}=\sigma_{e}+\epsilon^{2} A \sigma_{e} L\left[a^{\prime \prime}-a^{\prime}\right] \omega_{t}\left(a^{\prime \prime}\right)+O\left(\epsilon^{3}\right) .
$$

Substituting this expression into (A6) yields the formula for the risk-free rate.

\section{Proof of Proposition 3.5}

Using the expression in (A7) and the fact that the leverage constraint binds only when $\omega_{t}\left(a^{\prime}\right)\left(a^{\prime \prime}-a^{\prime}\right) \geq L$, we see that

$$
\sigma_{R t}^{c} \leq \sigma_{e}+\epsilon^{2} A \sigma_{e}\left[a^{\prime \prime}-a^{\prime}\right]^{2} \omega_{t}\left(a^{\prime}\right) \omega_{t}\left(a^{\prime \prime}\right)+O\left(\epsilon^{3}\right)
$$

Note that

$$
\begin{aligned}
\operatorname{var}[a] & =\left(a^{\prime}\right)^{2} \omega_{t}\left(a^{\prime}\right)+\left(a^{\prime \prime}\right)^{2} \omega_{t}\left(a^{\prime \prime}\right)-\left[a^{\prime} \omega_{t}\left(a^{\prime}\right)+a^{\prime \prime} \omega_{t}\left(a^{\prime \prime}\right)\right]^{2} \\
& =\left(a^{\prime}\right)^{2} \omega_{t}\left(a^{\prime}\right) \omega_{t}\left(a^{\prime \prime}\right)+\left(a^{\prime \prime}\right)^{2} \omega_{t}\left(a^{\prime}\right) \omega_{t}\left(a^{\prime \prime}\right)-2 a^{\prime} a^{\prime \prime} \omega_{t}\left(a^{\prime}\right) \omega_{t}\left(a^{\prime \prime}\right) \\
& =\left(a^{\prime \prime}-a^{\prime}\right)^{2} \omega_{t}\left(a^{\prime}\right) \omega_{t}\left(a^{\prime \prime}\right) .
\end{aligned}
$$

Therefore,

$$
\sigma_{R t}^{\mathrm{c}} \leq \sigma_{R t}^{\mathrm{u}}+O\left(\epsilon^{3}\right)
$$




\section{Proof of Proposition 3.6}

Assume that agents have risk aversion between $-\hat{a}$ to $+\hat{a}$, with a general wealth distribution, $\omega(a)$. Then,

$$
0=\int_{-\hat{a}}^{L-\phi_{1 t}}\left(b+\phi_{1 t}\right) \omega_{t}(a) d a+\int_{L-\phi_{1 t}}^{\hat{a}} L \omega_{t}(a) d a
$$

Differentiating the above with respect to $L$, we have:

$$
\begin{aligned}
0= & \int_{-\hat{a}}^{L-\phi_{1 t}} \frac{\partial \phi_{1 t}}{\partial L} \omega_{t}(a) d a+\left(L-\phi_{1 t}\right) \omega_{t}\left(L-\phi_{1 t}\right)\left(1-\frac{\partial \phi_{1 t}}{\partial L}\right) \\
& -L \omega_{t}\left(L-\phi_{1 t}\right)\left(1-\frac{\partial \phi_{1 t}}{\partial L}\right)+\int_{L-\phi_{1 t}}^{\hat{a}} \omega_{t}(a) d a
\end{aligned}
$$

which then implies that

$$
\frac{\partial \phi_{1 t}}{\partial L}=-\frac{\int_{L-\phi_{1 t}}^{\hat{a}} \omega_{t}(a) d a}{\int_{-\hat{a}}^{L-\phi_{1 t}} \omega_{t}(a) d a} \leq 0 .
$$

Since the first-order terms in expansions of the mean and the volatility of stock returns are not affected by the leverage constraint, this implies that asymptotically the interest rate in the constrained economy is lowered and the equity premium is increased by tightening the borrowing constraint.

Now, we compute the volatility of the returns process:

$$
d\left(\frac{P_{t}}{e_{t}}\right)=\epsilon \frac{A}{\rho} \int_{-\hat{a}}^{\hat{a}} a d \omega_{t}(a)+O\left(\epsilon^{3}\right)
$$

The wealth of agents for whom the constraint does not bind changes according to

$$
\begin{aligned}
\frac{d W_{t}(a)}{W_{t}(a)} & =[\ldots] d t+\left[1+\epsilon\left(a+\phi_{1 t}\right)\right] \sigma_{e} d Z_{t}+O\left(\epsilon^{2}\right), \\
\frac{d \omega_{t}(a)}{\omega_{t}(a)} & =[\ldots] d t+\epsilon\left(a+\phi_{1 t}\right) \sigma_{e} d Z_{t}+O\left(\epsilon^{2}\right) .
\end{aligned}
$$

For the constrained agents,

$$
\frac{d \omega_{t}(a)}{\omega_{t}(a)}=[\ldots] d t+\epsilon L \sigma_{e} d Z_{t}+O\left(\epsilon^{2}\right)
$$


so,

$$
d\left(\frac{P_{t}}{e_{t}}\right)=[\ldots] d t+\epsilon^{2} \frac{A \sigma_{e}}{\rho}\left[\int_{-\hat{a}}^{L-\phi_{1 t}} a \omega_{t}(a)\left(a+\phi_{1 t}\right) d a+\int_{L-\phi_{1 t}}^{\hat{a}} L a \omega_{t}(a) d a\right] d Z_{t}+O\left(\epsilon^{3}\right) .
$$

Thus,

$$
\begin{aligned}
\frac{\partial \sigma_{R t, 2}}{\partial L} & =\epsilon^{2} \frac{A \sigma_{e}}{\rho}\left[\int_{-\hat{a}}^{L-\phi_{1 t}} a \omega_{t}(a) \frac{\partial \phi_{1 t}}{\partial L} d a+\int_{L-\phi_{1 t}}^{\hat{a}} a \omega_{t}(a) d a\right] \\
& =\epsilon^{2} \frac{A \sigma_{e}}{\rho}[\int_{-\hat{a}}^{L-\phi_{1 t}} \underbrace{\left(a-L+\phi_{1 t}\right)}_{(-)} \omega_{t}(a) \underbrace{\frac{\partial \phi_{1 t}}{\partial L}}_{(-)} d a+\int_{L-\phi_{1 t}}^{\hat{a}} \underbrace{\left(a-L+\phi_{1 t}\right)}_{(+)} \omega_{t}(a) d a] \\
& \geq 0
\end{aligned}
$$

where $\sigma_{R t}=\sigma_{e}^{2}+\epsilon^{2} \sigma_{R t, 2}+O\left(\epsilon^{3}\right)$ and we have used (A8) to establish the second equality and to determine the sign of $\partial \phi_{1 t} / \partial L$. 
Figure 1: Volatility of stock returns in a heterogeneous economy

The ratio of the conditional volatility of stock returns to the volatility of the endowment process, $\sigma_{R t} / \sigma_{e}$, is plotted against the cross-sectional variance of portfolio holdings, $\operatorname{var}_{a}[\pi(a)]$. The solid line corresponds to the analytical asymptotic solution, the dashed line is computed numerically. The following parameter values are used: $\mu_{e}=.02, \sigma_{e}=.03, \rho=.02$. There are two types of agents in the economy, $a^{\prime}=0$ and $a^{\prime \prime}=1$. The small parameter $\epsilon$ takes values of $.5,-.5,-1$, and -4 .
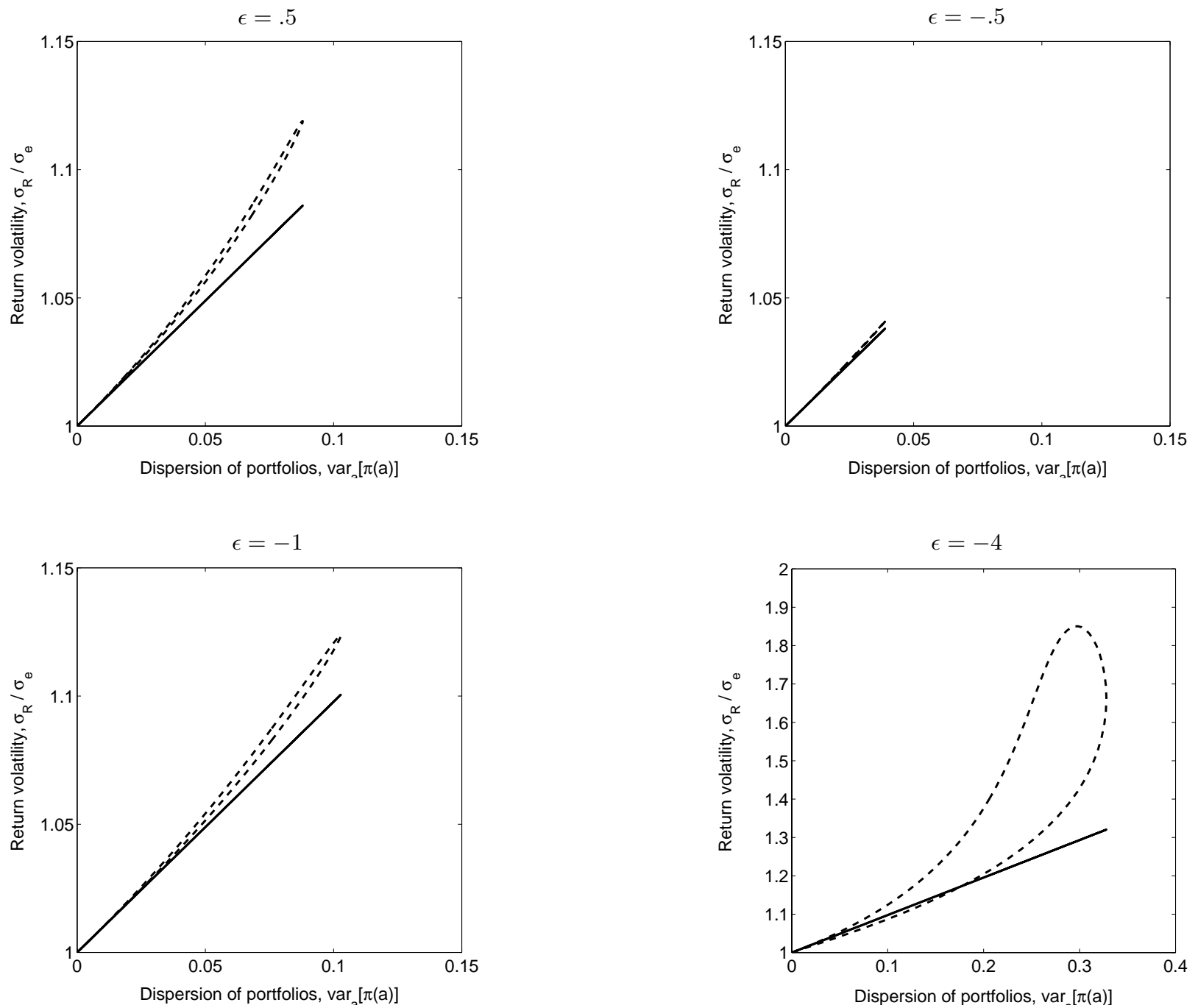


\section{References}

Campbell, John Y., 1993, Intertemporal asset pricing without consumption data, American Economic Review 83, 487-512.

Campbell, John Y. and Luis M. Viceira, 1999, Consumption and portfolio decisions when expected returns are time varying, Quarterly Journal of Economics 114, 433-495.

Chan, Yeung Lewis and Leonid Kogan, 2000, Heterogeneous preferences, catching up with the Joneses and the dynamics of asset prices, Working paper, U. of Pennsylvania.

Cox, John C. and Chi-fu Huang, 1989, Optional consumption and portfolio policies when assets prices follow a diffusion process, Journal of Economic Theory 49, 33-83.

Cuoco, Domenico, 1997, Optimal consumption and equilibrium prices with portfolio constraints and stochastic income, Journal of Economic Theory 72, 33-73.

Cuoco, Domenico, and Hua He, 1994a, Dynamic aggregation and computation of equilibria in finite-dimensional economies with incomplete financial markets, Working paper, University of Pennsylvania.

Cuoco, Domenico and Hua He, 1994b, Dynamic equilibrium in infinite-dimensional economies with incomplete financial markets, Working paper, University of Pennsylvania.

Cvitanić, Jakša and Ioannis Karatzas, 1992, Convex duality in constrained portfolio optimization, Annals of Applied Probability 2, 767-818.

Den Haan, Wouter J. and Albert Marcet, 1994, Accuracy in simulations, The Review of Economic Studies 61.1, 3-17.

Detemple, Jerome and Shashidhar Murthy, 1997, Equilibrium asset prices and no-arbitrage with portfolio constraints, Review of Financial Studies 10.4, 1133-1174.

Duffie, Darrell and Larry G. Epstein, 1992, Stochastic Differential Utility, Econometrica, 60, 353394.

Dumas, Bernard, 1989, Two-person dynamic equilibrium in the capital market, Review of Financial Studies, 2, 157-188.

Grossman, Sanford J. and Jean-Luc Vila, 1992, Optimal dynamic trading with leverage constraints, Journal of Financial and Quantitative Analysis 27, 151-168.

Hakansson, Nils H., 1971, On optimal myopic portfolio policies with and without serial correlation of yields, Journal of Business 44, 324-334.

Heaton, John and Deborah Lucas, 1996, Evaluating the effects of incomplete markets on risk sharing and asset pricing, Journal of Political Economy 104(3), 443-487.

Judd, Kenneth L., 1996, Approximation, perturbation, and projection methods for economic growth models, in H. Amman, D. Kendrick, and J. Rust, eds., Handbook of Computational Economics, Elsevier, Amsterdam. 
Judd, Kenneth L., 1998, Numerical Methods in Economics, MIT Press, Cambridge, MA.

Kim, Tong Suk and Edward Omberg, 1996, Dynamic nonmyopic portfolio behavior, Review of Financial Studies 9, 141-161.

Kreps, David and E. Porteus, 1978, Temporal resolution of uncertainty and dynamic choice theory," Econometrica, 46, 185-200.

Leland, Hayne (1968), Dynamic portfolio theory, Ph.D. thesis, Harvard University.

Liu, Jun, 1998, Portfolio selection in stochastic environments, Working paper, Stanford University.

Marcet, Albert and Kenneth Singleton, 1999, Equilibrium asset prices and savings of heterogeneous agents in the presence of incomplete markets and portfolio constraints, Macroeconomic Dynamics, forthcoming.

Merton, Robert C., 1969, Lifetime portfolio selection under uncertainty: The continuous time case, Review of Economics and Statistics 51, 247-257.

Merton, Robert C., 1971, Optimum consumption and portfolio rules in a continuous time model, Journal of Economic Theory 3, 373-413.

Mossin, Jan, 1968, Optimal multiperiod portfolio policies, Journal of Business 41, 215-229.

Wachter, Jessica, 1998, Portfolio and consumption decisions under mean-reverting returns: An exact solution for complete markets, Working paper, Harvard University.

Wang, Jiang, 1996, The term structure of interest rates in a pure exchange economy with heterogeneous investors , Journal of Financial Economics 41.1,75-110. 